\title{
Seletividade de Herbicidas Aplicados na Grama Batatais e na Grama SÃo Carlos ${ }^{1}$
}

\author{
Selectivity of Herbicides Applied on Bahiagrass and Broadleaf Carpetgrass Turfs
}

\author{
COSTA, N.V. ${ }^{2}$, MARTINS, D. ${ }^{3}$, RODRIGUES, A.C.P. ${ }^{4}$ e CARDOSO, L.A. ${ }^{4}$
}

\begin{abstract}
RESUMO - Objetivou-se avaliar a seletividade de herbicidas aplicados na grama Batatais (Paspalum notatum) e na grama São Carlos (Axonopus compressus) em campo. O delineamento experimental utilizado foi o de blocos casualizados com quatro repetições. As gramas foram cortadas a $3 \mathrm{~cm}$ de altura e, em seguida, realizaram-se as aplicações dos herbicidas. Os tratamentos utilizados foram: testemunha (sem aplicação), fluazifop-p-butil (125 g ha-1), sethoxydim+óleo mineral (276 $\mathrm{g} \mathrm{ha}^{-1}+0,5 \% \mathrm{v} \mathrm{v}^{-1}$ de Assist), bispyribac-sodium $\left(25 \mathrm{~g} \mathrm{ha}^{-1}\right)$, chlorimuron-ethyl (15 $\left.\mathrm{g} \mathrm{ha}^{-1}\right)$, ethoxysulfuron (150 $\left.\mathrm{g} \mathrm{ha}^{-1}\right)$, halosulfuron $\left(112,5 \mathrm{~g} \mathrm{ha}^{-1}\right)$, iodosulfuron-methyl (10 $\left.\mathrm{g} \mathrm{ha}^{-1}\right)$, metsulfuron-methyl $\left(2,4 \mathrm{~g} \mathrm{ha}^{-1}\right)$, nicosulfuron $\left(125 \mathrm{~g} \mathrm{ha}^{-1}\right)$, pyrithiobac-sodium (140 $\left.\mathrm{g} \mathrm{ha}^{-1}\right)$, trifloxysulfuron-sodium $\left(22,5 \mathrm{~g} \mathrm{ha}^{-1}\right), 2,4-\mathrm{D}\left(720 \mathrm{~g} \mathrm{ha}^{-1}\right)$, quinclorac (375 $\left.\mathrm{g} \mathrm{ha}^{-1}\right)$, atrazina $\left(1.250 \mathrm{~g} \mathrm{ha}^{-1}\right)$, bentazon $\left(600 \mathrm{~g} \mathrm{ha}^{-1}\right)$, linuron $\left(1.350 \mathrm{~g} \mathrm{ha}^{-1}\right)$, fomesafen $\left(187,5 \mathrm{~g} \mathrm{ha}^{-1}\right)$, lactofen $\left(120 \mathrm{~g} \mathrm{ha}^{-1}\right)$, oxadiazon $\left(600 \mathrm{~g} \mathrm{ha}^{-1}\right)$ e oxyfluorfen $\left(720 \mathrm{~g} \mathrm{ha}^{-1}\right)$. Os herbicidas que apresentaram potencial de seletividade para o gramado de $P$. notatum foram o chlorimuron-ethyl, ethoxysulfuron, pyrithiobac-sodium, 2,4-D, bentazon e fomesafen; já para o gramado de A. compressus foram o chlorimuron-ethyl, ethoxysulfuron, halosulfuron, iodosulfuron-methyl, metsulfuron-methyl, pyrithiobac-sodium, 2,4-D, quinclorac, atrazina, bentazon, além do fomesafen.
\end{abstract}

Palavras-chave: gramado, planta daninha, Paspalum notatum, Axonopus compressus.

\begin{abstract}
The objective of this study was to evaluate the selectivity of herbicides applied on Bahiagrass (Paspalum notatum) and Broadleaf Carpetgrass (Axonopus compressus) turfgrasses under field conditions. The experiment was arranged in a randomized block design, with four replications. The turfgrasses were cut to the height of $3 \mathrm{~cm}$, followed by herbicide application.. The

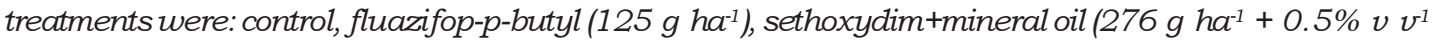
of Assist), bispyribac-sodium (25 $\left.\mathrm{g} \mathrm{ha} \mathrm{a}^{-1}\right)$, chlorimuron-ethyl (15 $\left.\mathrm{g} \mathrm{ha} \mathrm{a}^{-1}\right)$, ethoxysulfuron (150 $\mathrm{g} \mathrm{ha^{-1 }}$ ), halosulfuron (112.5 $\left.\mathrm{g} \mathrm{ha}^{-1}\right)$, iodosulfuron-methyl (10 g ha-1), metsulfuron-methyl $\left(2,4 \mathrm{~g} \mathrm{ha}^{-1}\right)$,

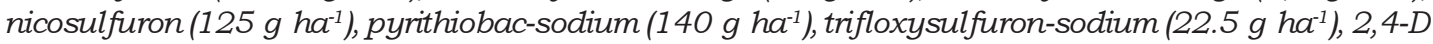
$\left(720 \mathrm{~g} \mathrm{ha}^{-1}\right)$, quinclorac $\left(375 \mathrm{~g} \mathrm{ha} \mathrm{f}^{-1}\right)$, atrazine $(1,250 \mathrm{~g} \mathrm{ha})$, bentazon $\left(600 \mathrm{~g} \mathrm{ha} \mathrm{a}^{-1}\right)$, linuron $(1,350 \mathrm{~g} \mathrm{ha}-1)$, fomesafen (187.5 $\left.\mathrm{g} \mathrm{ha}^{-1}\right)$, lactofen (120 $\left.\mathrm{g} \mathrm{ha}^{-1}\right)$, oxadiazon $\left(600 \mathrm{~g} \mathrm{ha}^{-1}\right)$ and oxyfluorfen (720 $\mathrm{g} \mathrm{ha}$ ). The herbicides with selectivity potential for $\boldsymbol{P}$. notatum were chlorimuron-ethyl, ethoxysulfuron, pyrithiobac-sodium, 2.4-D, bentazon and fomesafem and those with selectivity potential for A. compressus were chlorimuron-ethyl, ethoxysulfuron, halosulfuron, iodosulfuron-methyl, metsulfuron-methyl, pyrithiobac-sodium, 2.4-D, quinclorac, atrazine and bentazon, besides fomesafen.
\end{abstract}

Keywords: turfgrass, weed, Paspalum notatum, Axonopus compressus.

Recebido para publicação em 28.3.2009 e na forma revisada em 15.6.2010.

2 Professor Adjunto da Unioeste/CCA, Marechal Cândido Rondon-PR, <neumarciovc@hotmail.com>; ${ }^{3}$ Professor Adjunto do Dep. de Produção Vegetal - FCA/UNESP, Fazenda Lageado, Caixa Postal 237, 18603-970 Botucatu-SP; ${ }^{4}$ Doutorando em Agronomia do Dep. de Produção Vegetal, FCA/UNESP, Botucatu-SP. 


\section{INTRODUÇÃO}

No mercado brasileiro, a produção de grama está classificada em duas categorias: a de gramas cultivadas e a de nativas. Assim, como principais exemplos de gramas nativas no Brasil destacam-se a grama Batatais (Paspalum notatum) e a grama São Carlos (Axonopus compressus). Essas espécies podem ser utilizadas em obras públicas, parques industriais, áreas esportivas, assim como em áreas residenciais. Contudo, a interferência das plantas daninhas pode prejudicar a formação, o manejo e a estética dos gramados, além de elas competirem por água, luz, nutrientes e espaço físico, chegando em alguns casos a dizimá-los por completo (Busey, 2003).

A introdução das plantas daninhas ocorre na maioria das vezes no ato da implantação do gramado, com a utilização de solo e de mudas normalmente originárias de áreas com a presença de diversas espécies de plantas daninhas. Além disso, a degradação do gramado, em função da compactação do solo e de cortes sucessivos, com a retirada da grama aparada sem a devida reposição dos nutrientes, pode levar à predominância de plantas daninhas (Freitas et al., 2003).

Apesar de os gramados tolerarem a competição das plantas daninhas em baixos niveis durante o seu estabelecimento, a utilização de herbicidas frequentemente torna-se necessária para a manutenção do controle das espécies daninhas (Johnson \& Carrow, 1999) e prolongar a vida útil do gramado. É importante ressaltar que o uso de herbicidas não seletivos pode provocar injúrias aos gramados e dificultar o seu estabelecimento na área, diminuindo a competição com as plantas daninhas (Fagerness et al., 2002).

Em outros países, vários pesquisadores destacaram a viabilidade do uso de herbicidas aplicados em pré e pós-emergência em vários tipos de gramados, a exemplo de atrazina, oxadiazon, quinclorac, sethoxydim, entre outros, bem como a possibilidade da utilização desses produtos em mistura em tanque, de forma a maximizar os efeitos dos diferentes mecanismos de ação no controle das plantas daninhas (Turner et al., 1990; McCarty et al., 1995; Johnson \& Carrow, 1999; Brecke et al., 2001; Fagerness et al., 2002).
Contudo, Christoffoleti \& Aranda (2001) ressaltaram que não existe regra única de recomendação de herbicidas, sendo, portanto, necessárias recomendações diferenciadas, dependendo do nivel de toxicidade tolerado pelo gramado. Do mesmo modo, Busey (2003) e Busey \& Johnston (2006) destacaram a importância do manejo integrado das plantas daninhas em gramados; para seu sucesso, devem-se identificar detalhadamente os efeitos das interações dos manejos cultural e químico, uma vez que essas práticas podem ser complementares, além de proporcionar maior flexibilidade para as tomadas de decisão e exercer menor pressão de seleção sobre as plantas daninhas.

Dessa maneira, a identificação de herbicidas seletivos e que apresentem mecanismos de ação distintos torna-se fundamental para o desenvolvimento de programas de manejo das plantas daninhas em gramados para as condições brasileiras, além de evitar a seleção de espécies resistentes a herbicidas. Rodrigues $\&$ Almeida (2005) afirmam que no Brasil apenas o herbicida 2,4-D é registrado para utilização em gramados.

Assim, o objetivo do presente trabalho foi o de avaliar a seletividade de diferentes herbicidas aplicados na grama Batatais ( $P$. notatum) e na grama São Carlos (A. compressus) em condições de campo.

\section{MATERIAL E MÉTODOS}

O estudo foi realizado no Núcleo de Pesquisas Avançadas em Matologia - NUPAM, do Departamento de Produção Vegetal, pertencente à Faculdade de Ciências Agronômicas de Botucatu/UNESP. O local do experimento apresenta as seguintes coordenadas geográficas: latitude $22^{\circ} 07^{\prime} 56^{\prime \prime} \mathrm{S}$, longitude 746'ㅇ' 'W Gr. e altitude de $762 \mathrm{~m}$. Foram avaliadas as espécies grama Batatais $(P$. notatum) e grama São Carlos (A. compressus), as quais foram plantadas em tabuleiros individuais e estavam com 26 meses de idade no momento da aplicação dos tratamentos. Para cada espécie foi utilizado o delineamento experimental em blocos casualizados com quatro repetições, sendo as parcelas constituídas de $2 \mathrm{~m}$ de largura por $2 \mathrm{~m}$ de comprimento. $\mathrm{Na}$ Tabela 1 encontram-se os herbicidas avaliados 
por nome comum, mecanismo de ação e dose utilizada.

Em 12/1/2006, os gramados foram cortados à altura de $3 \mathrm{~cm}$ com auxílio de um aparador de grama motorizado e, após dois dias, foram realizadas as aplicações dos tratamentos, no período da manhã, em condições de temperatura de $27^{\circ} \mathrm{C}$, solo úmido e umidade relativa do ar em torno de $64 \%$. Foi utilizado um pulverizador costal, pressurizado a $\mathrm{CO}_{2} \mathrm{e}$ munido de barra com quatro pontas de jato plano DG $110.02 \mathrm{VS}$, espaçados entre si de $50 \mathrm{~cm}$, sendo o consumo de calda equivalente a $200 \mathrm{~L} \mathrm{ha}^{-1}$. Durante a aplicação, as parcelas foram protegidas lateralmente com biombos de lona plástica, com o objetivo de evitar deriva de calda pulverizada para as parcelas adjacentes.

As avaliações de intoxicação nos gramados foram feitas de forma visual, utilizando-se uma escala de percentual de notas, em que 0 (zero) corresponde a nenhuma injúria demonstrada pela planta e 100 (cem) à morte das plantas (SBCPD, 1995). Dessa forma, para evitar

Tabela 1 - Herbicidas avaliados, indicados por nome comum, mecanismo de ação e dose utilizada. Botucatu-SP, 2006

\begin{tabular}{|l|c|c|}
\hline \multirow{2}{*}{ Nome comum } & Mecanismo de & Dose \\
\cline { 3 - 3 } & ação & $\left(\mathrm{g}\right.$ i.a. ha $\left.^{-1}\right)$ \\
\hline Testemunha & --- & --- \\
\hline fluazifop-p-butil & ACCase & 125,0 \\
\hline sethoxydim ${ }^{1 /}$ & ACCase & 276,0 \\
\hline bispyribac-sodium & ALS & 25,0 \\
\hline chlorimuron-ethyl & ALS & 15,0 \\
\hline ethoxysulfuron & ALS & 150,0 \\
\hline halosulfuron & ALS & 112,5 \\
\hline iodosulfuron-methyl & ALS & 10,0 \\
\hline metsulfuron-methyl & ALS & 2,4 \\
\hline nicosulfuron & ALS & 40,0 \\
\hline pyrithiobac-sodium & ALS & 140,0 \\
\hline trifloxysulfuron-sodium & ALS & 22,5 \\
\hline 2,4-D & Auxina & 720,0 \\
\hline quinclorac & Auxina & 375,0 \\
\hline atrazina & Fotossistema II & $1.250,0$ \\
\hline bentazon & Fotossistema II & 600,0 \\
\hline linuron & Fotossistema II & $1.350,0$ \\
\hline fomesafen & Protox & 187,5 \\
\hline lactofen & Protox & 120,0 \\
\hline oxadiazon & Protox & 600,0 \\
\hline oxyfluorfen & Protox & 720,0 \\
\hline
\end{tabular}

${ }^{1 /}$ Adicionou-se o adjuvante Assist $\left(0,5 \% \mathrm{v} \mathrm{v}^{-1}\right)$ à calda de aplicação. grandes prejuízos ao aspecto visual do gramado e facilitar a interpretação dos resultados de intoxicação, consideraram-se como sintomas leves as notas inferiores a $10,0 \%$; como moderados, as notas entre 10,1 e $20,0 \%$; e como severos e não aceitáveis esteticamente, as notas superiores a $20,0 \%$. As avaliações foram realizadas aos 3, 7, 14, 26 e 49 dias após aplicação dos herbicidas. Os parâmetros utilizados para o estabelecimento das notas foram inibição do crescimento, quantidade e uniformidade das injúrias, capacidade de rebrota das plantas e quantidade de plantas mortas.

Os caracteres morfológicos avaliados, aos 49 dias após a aplicação dos herbicidas (DAA), foram número e comprimento de inflorescência, altura da grama e massa seca da parte aérea.

O número de inflorescências foi avaliado por amostragem, com auxílio de um quadro de $0,25 \mathrm{~m}^{2}$ colocado no centro das parcelas, e o comprimento das inflorescências foi determinado com auxílio de uma régua graduada, medindo-se a distância entre o solo e as extremidades, amostrando-se apenas a inflorescência mais alta do centro de cada parcela. As avaliações da altura da grama foram realizadas com auxílio de duas réguas graduadas, sendo posicionadas, no momento da avaliação, perpendicular e horizontalmente ao solo (em forma de cruz) em apenas um ponto de cada parcela, medindo-se a distância entre o solo e a extremidade da folha mais alta. A régua na horizontal tinha como função facilitar a determinação da folha mais alta do centro da parcela.

Determinou-se a massa seca da parte aérea produzida pelas gramas, coletando-se a massa vegetal produzida na parcela após o corte da grama a $3 \mathrm{~cm}$ de altura, com auxílio de um aparador de grama motorizado, para posterior secagem em estufa de circulação forçada de ar por 72 horas a $65^{\circ} \mathrm{C}$.

Os resultados foram submetidos à análise de variância, empregando-se o teste de média LSD a $5 \%$ de probabilidade. Os dados originais em número de inflorescências e porcentagem de intoxicação foram transformados utilizando-se $(x+0,5)^{0,5}$ e arco seno $(x / 100)^{0,5}$, respectivamente. 


\section{RESULTADOS E DISCUSSÃO}

Na Tabela 2 são apresentadas as porcentagens de intoxicação em plantas de $P$. notatum após a aplicação de diferentes herbicidas. Verificou-se que todos eles promoveram intoxicação nas plantas a partir de 3 DAA, sendo os sintomas mais severos encontrados nas parcelas tratadas com oxyfluorfen, com média de $22,5 \%$.

Analisando os herbicidas por mecanismo de ação, observou-se que para os inibidores da ACCase (acetil-coenzima-A carboxilase) os sintomas foram considerados severos a partir de 7 DAA; aos 14 DAA, os herbicidas fluazifop-pbutil e sethoxydim proporcionaram intoxicação de 90,0 e 74,3\%, respectivamente. Entretanto, Baker et al. (1999), avaliando a aplicação dos herbicidas fluazifop-p-butil (110 $\left.\mathrm{g} \mathrm{ha}^{-1}\right)$ e sethoxydim (560 $\left.\mathrm{g} \mathrm{ha}^{-1}\right)$ em $P$. notatum por três anos consecutivos, não verificaram prejuízos no aspecto visual da grama por um período de até 84 DAA em cada ano.
Para os inibidores da ALS (acetolactato sintase) chlorimuron-ethyl e ethoxysulfuron, os sintomas foram considerados leves, persistindo por no máximo 14 DAA, enquanto para pyrithiobac-sodium eles foram moderados. Dessa forma, esses níveis de intoxicação podem ser tolerados em gramados com $P$. notatum, uma vez que ocorreram por periodo curto de tempo. A utilização de irrigação, fertilizante ou de corantes artificiais pode contribuir para melhorar o aspecto visual do gramado em situações de moderada toxicidade por herbicidas, além de reduzir o período do desaparecimento dos sintomas de intoxicação.

O halosulfuron e o metsulfuron-methyl apresentaram sintomas severos de intoxicação aos 7 DAA; após 14 DAA, os sintomas regrediram para níveis leves, desaparecendo completamente aos 26 DAA. Entretanto, quando se utilizaram bispyribac-sodium, iodosulfuron-methyl, nicosulfuron ou trifloxysulfuron-sodium, as plantas de $P$. notatum apresentaram intoxicação severa $(>20,0 \%)$,

Tabela 2 - Porcentagem de intoxicação em plantas de P. notatum após a aplicação de diferentes herbicidas. Botucatu-SP, 2006

\begin{tabular}{|c|c|c|c|c|c|c|c|}
\hline \multirow{2}{*}{ Tratamento } & \multirow{2}{*}{$\frac{\text { Dose }}{\left(\mathrm{g} \text { i.a. } \mathrm{ha}^{-1}\right)}$} & \multirow{2}{*}{$\begin{array}{c}\text { Mecanismo } \\
\text { de ação }\end{array}$} & \multicolumn{5}{|c|}{ Dias após a aplicação ${ }^{\underline{2}}$} \\
\hline & & & 3 & 7 & 14 & 26 & 49 \\
\hline Testemunha & --- & --- & $0,0(0,0) \mathrm{h}$ & $0,0(0,0) \mathrm{h}$ & $0,0(0,0) \mathrm{k}$ & $0,0(0,0) \mathrm{d}$ & 0,0 \\
\hline fluazifop-p-butil & 125,0 & ACCase & $6,5(14,5) b$ & $70,0(57,3) \mathrm{a}$ & $90,0(71,9) \mathrm{a}$ & $67,5(55,5) a$ & 0,0 \\
\hline sethoxydim$^{\underline{1}}$ & 276,0 & ACCase & $4,5(12,2)$ bcd & $63,8(53,5) a$ & $74,3(60,5) \mathrm{bc}$ & $5,0(9,2) \mathrm{cd}$ & 0,0 \\
\hline bispyribac-sodium & 25,0 & ALS & $5,0(12,9) \mathrm{bc}$ & $63,8(53,3) a b$ & $77,5(61,8) b$ & $37,5(37,6) b$ & 0,0 \\
\hline chlorimuron-ethyl & 15,0 & ALS & $4,0(11,0)$ bcde & $8,0(16,3) \mathrm{g}$ & $1,0(4,1) \mathrm{jk}$ & $0,0(0,0) \mathrm{d}$ & 0,0 \\
\hline ethoxysulfuron & 150,0 & ALS & $2,3(8,4)$ efg & $10,0(17,2) \mathrm{g}$ & $0,8(3,5) \mathrm{k}$ & $0,0(0,0) \mathrm{d}$ & 0,0 \\
\hline halosulfuron & 112,5 & ALS & $3,8(11,0)$ bcde & $37,5(37,3)$ cde & $8,0(15,6)$ hi & $0,0(0,0) \mathrm{d}$ & 0,0 \\
\hline iodosulfuron-methyl & 10,0 & ALS & $4,5(12,2)$ bcd & $38,8(38,4)$ cde & $40,0(39,1) \mathrm{d}$ & $70,0(57,2) a$ & 0,0 \\
\hline metsulfuron-methyl & 2,4 & ALS & $2,8(9,3)$ cdef & $28,8(31,5)$ def & $10,0(18,1) \mathrm{fgh}$ & $0,0(0,0) \mathrm{d}$ & 0,0 \\
\hline nicosulfuron & 40,0 & ALS & $4,0(11,5)$ bcde & $57,5(49,7)$ abc & $63,8(53,5) \mathrm{c}$ & $63,8(53,4) a$ & 0,0 \\
\hline pyrithiobac-sodium & 140,0 & ALS & $4,8(12,5) \mathrm{bc}$ & $20,0(26,5)$ defg & $16,3(23,6)$ ef & $0,0(0,0) \mathrm{d}$ & 0,0 \\
\hline trifloxysulfuron-sodium & 22,5 & ALS & $3,8(11,1)$ bcde & $41,3(39,9)$ bcd & $45,0(42,1) \mathrm{d}$ & $75,0(60,7) a$ & 0,0 \\
\hline $2,4-\mathrm{D}$ & 720,0 & Auxina & $2,8(9,2)$ cdef & $0,0(0,0) \mathrm{h}$ & $0,0(0,0) \mathrm{k}$ & $0,0(0,0) \mathrm{d}$ & 0,0 \\
\hline quinclorac & 375,0 & Auxina & $3,3(10,3)$ cde & $24,5(28,1)$ defg & $40,0(39,2) \mathrm{d}$ & $41,3(39,8) b$ & 0,0 \\
\hline atrazina & $1.250,0$ & Foto II & $1,3(5,4) \mathrm{g}$ & $21,3(26,2)$ efg & $22,5(28,3) \mathrm{e}$ & $12,5(17,5) \mathrm{c}$ & 0,0 \\
\hline bentazon & 600,0 & Foto II & $1,5(6,1) \mathrm{fg}$ & $0,0(0,0) \mathrm{h}$ & $0,0(0,0) \mathrm{k}$ & $0,0(0,0) \mathrm{d}$ & 0,0 \\
\hline linuron & $1.350,0$ & Foto II & $2,3(8,5)$ defg & $25,0(29,1)$ defg & $16,3(23,6)$ ef & $1,3(3,2) \mathrm{d}$ & 0,0 \\
\hline fomesafen & 187,5 & Protox & $3,8(11,0)$ bcde & $18,8(22,0) \mathrm{fg}$ & $4,3(11,7) \mathrm{ij}$ & $0,0(0,0) \mathrm{d}$ & 0,0 \\
\hline lactofen & 120,0 & Protox & $3,3(10,2)$ cde & $28,8(31,3)$ def & $6,3(14,3) \mathrm{i}$ & $0,0(0,0) \mathrm{d}$ & 0,0 \\
\hline oxadiazon & 600,0 & Protox & $5,3(12,7)$ bc & $32,5(33,8)$ def & $8,8(17,0)$ fgh & $0,0(0,0) \mathrm{d}$ & 0,0 \\
\hline oxyfluorfen & 720,0 & Protox & $22,5(28,2) a$ & $55,0(47,9)$ abc & $15,0(22,7)$ efg & $0,0(0,0) \mathrm{d}$ & 0,0 \\
\hline $\mathrm{F}_{\text {Tratamento }}$ & & & $14,401^{* *}$ & $13,190 * *$ & $62,530 * *$ & $49,239 * *$ & -- \\
\hline $\mathrm{F}_{\text {Bloco }}$ & & & $1,511^{\mathrm{ns}}$ & $4,604 * *$ & $0,439^{\mathrm{ns}}$ & $2,360^{\mathrm{ns}}$ & - \\
\hline $\mathrm{CV}(\%)$ & & & 24,68 & 31,17 & 21,12 & 41,96 & - \\
\hline d.m.s. & & & 3,786 & 13,415 & 7,826 & 9,439 & --- \\
\hline
\end{tabular}

$\underline{1}$ / Adicionou-se o adjuvante Assist $\left(0,5 \% \mathrm{v} \mathrm{v}^{-1}\right)$ à calda de aplicação. ${ }^{2 /}$ dados originais, entre parênteses, foram transformados em arco seno seno $(\mathrm{x} / 100)^{0,5}$. Médias seguidas de mesma letra, nas linhas, não diferem estatisticamente entre si a $5 \%$ de probabilidade pelo teste LSD. ** significativo a $1 \%$ de probabilidade; * significativo a $5 \%$ de probabilidade; ${ }^{n s}$ não significativo. 
prejudicando o aspecto visual do gramado até 26 DAA (Tabela 2).

Entre os mimetizadores de auxina, 2,4-D destacou-se entre os herbicidas que apresentaram as menores médias de intoxicação $(\leq 2,8 \%)$, sendo estas verificadas somente até 3 DAA, enquanto o quinclorac proporcionou sintomas severos de $41,3 \%$ aos 26 DAA. Quanto aos inibidores do fotossistema II, apenas o bentazon mostrou sintomas leves de intoxicação - verificada somente até 3 DAA. Contudo, atrazina e linuron proporcionaram sintomas severos a partir de 7 DAA, regredindo para moderados e leves, respectivamente, aos 26 DAA.

Da mesma forma, para os inibidores da Protox (protoporfirinogênio oxidase), fomesafen, lactofen, oxadiazon e oxyfluorfen, os valores mais elevados de intoxicação foram observados aos 7 DAA, sendo considerados de moderados a severos, porém, com exceção do oxyfluorfen, os sintomas regrediram para leve aos 14 DAA.

No geral, os herbicidas chlorimuron-ethyl, ethoxysulfuron, pyrithiobac-sodium, 2,4-D, bentazon e fomesafen foram os que apresentaram sintomas de intoxicação leves a moderados por no máximo até 14 DAA; a partir dessa data os sintomas desapareceram completamente. Por outro lado, os herbicidas fluazifopp-butil, bispyribac-sodium, iodosulfuronmethyl, nicosulfuron, trifloxysulfuron-sodium e quinclorac promoveram intoxicações severas por até 26 DAA.

Ressalta-se que aos 49 DAA a grama de $P$. notatum não apresentou sintomas de intoxicação após a aplicação dos herbicidas avaliados.

Pode-se verificar que, para a densidade de inflorescências, apenas os herbicidas fluazifop-p-butil, nicosulfuron e trifloxysulfuron-sodium promoveram redução de 100,0\% em relação à testemunha. Entretanto, os herbicidas sethoxydim, bispyribac-sodium e iodosulfuron-methyl reduziram a densidade de inflorescências em torno de 65,4, 76,0 e 96,2\%, respectivamente (Tabela 3 ).

Baker et al. (1999) observaram que a aplicação dos herbicidas fluazifop-p-butil (110 $\left.\mathrm{g} \mathrm{ha}^{-1}\right)$ e do sethoxydim (560 $\left.\mathrm{g} \mathrm{ha}^{-1}\right) \mathrm{em}$
$P$. notatum por três anos consecutivos apresentou resultados variáveis com relação à supressão da emissão das inflorescências; contudo, esses herbicidas podem reduzir em cerca de 100,0 e 70,0\%, respectivamente, essa característica.

No tocante aos dados da altura das inflorescências, observou-se comportamento semelhante ao de densidade de inflorescências. $O$ fluazifop-p-butil, nicosulfuron e trifloxysulfuron-sodium promoveram redução de 100,0\% em relação à testemunha. Os herbicidas sethoxydim, bispyribac-sodium, iodosulfuronmethyl e atrazina promoveram reduções na altura das inflorescências na ordem de 32,1 ; 30,$5 ; 67,0 ;$ e $33,0 \%$, respectivamente, em relação à testemunha.

Com relação à altura da grama, notou-se que somente os inibidores da ALS nicosulfuron e trifloxysulfuron-sodium reduziram o crescimento do gramado em torno de 35,1 e 39,7\%, respectivamente, aos 49 DAA.

Do mesmo modo, verificou-se que os inibidores da ALS bispyribac-sodium, halosulfuron, iodosulfuron-methyl, nicosulfuron e trifloxysulfuron-sodium reduziram a massa seca da parte aérea da grama em torno de 40,1; 31,4; 55,3; 72,9; e 65,9\% em relação à testemunha, aos 49 DAA. Ressalta-se que, com exceção do halosulfuron, esses herbicidas proporcionaram sintomas severos de intoxicação no gramado por até 26 DAA; assim, esse fato pode explicar a redução da massa seca proporcionada por esses herbicidas na grama $P$. notatum.

Apesar de o fluazifop-p-butil e o quinclorac causarem sintomas severos por até 26 DAA em $P$. notatum, esse fato não se refletiu em redução da massa seca da parte aérea do gramado (Tabelas 2 e 3 ).

Considerando os herbicidas que proporcionaram intoxicação leve a moderada por no máximo 14 DAA e que não reduziram significativamente a massa seca da parte aérea da grama $P$. notatum por até 49 DAA, destacaram-se chlorimuron-ethyl, ethoxysulfuron, pyrithiobac-sodium, 2,4-D, bentazon e fomesafen.

Da mesma forma, os herbicidas fluazifopp-butil, sethoxydim, metsulfuron-methyl, quinclorac, atrazina, linuron, lactofen,

Planta Daninha, Viçosa-MG, v. 28, n. 2, p. 365-374, 2010 
Tabela 3 - Valores médios de número de inflorescência, altura da planta, altura de inflorescência e massa seca do gramado de $P$. notatum aos 49 dias após a aplicação de diferentes herbicidas. Botucatu-SP, 2006

\begin{tabular}{|c|c|c|c|c|c|c|}
\hline \multirow[t]{2}{*}{ Tratamento } & Dose & \multirow{2}{*}{$\begin{array}{l}\text { Mecanismo } \\
\text { de ação }\end{array}$} & $\begin{array}{l}\text { Densidade de } \\
\text { Inflorescência }\end{array}$ & $\begin{array}{c}\text { Altura da } \\
\text { Inflorescência }\end{array}$ & $\begin{array}{c}\text { Altura da } \\
\text { grama }\end{array}$ & \multirow{2}{*}{$\frac{\text { Massa seca }}{\left(\mathrm{kg} \mathrm{ha}^{-1}\right)}$} \\
\hline & $\left(\right.$ g i.a. ha $\left.{ }^{-1}\right)$ & & $\left(\text { número } \mathrm{m}^{-2}\right)^{2 / 1}$ & \multicolumn{2}{|c|}{$(\mathrm{cm})$} & \\
\hline Testemunha & --- & --- & $104,0(10,2) \mathrm{ab}$ & $31,5 \mathrm{ab}$ & $13,1 \mathrm{ab}$ & $1551,5 \mathrm{ab}$ \\
\hline fluazifop-p-butil & 125,0 & ACCase & $0,0(0,7) \mathrm{f}$ & $0,0 \mathrm{~g}$ & $13,5 \mathrm{a}$ & $1456,7 \mathrm{abc}$ \\
\hline sethoxydim $^{\frac{1}{1}}$ & 276,0 & ACCase & $36,0(5,8) \mathrm{cd}$ & $21,4 \mathrm{de}$ & 11,5 abcd & 1352,1 abcd \\
\hline bispyribac-sodium & 25,0 & ALS & $25,0(4,9) \mathrm{de}$ & $21,9 \mathrm{cde}$ & 11,5 abcd & 928,9 def \\
\hline chlorimuron-ethyl & 15,0 & ALS & $151,0(12,2) \mathrm{a}$ & $36,5 \mathrm{a}$ & $13,0 \mathrm{ab}$ & $1471,9 \mathrm{abc}$ \\
\hline ethoxysulfuron & 150,0 & ALS & $130,0(11,3) \mathrm{ab}$ & $32,6 \mathrm{ab}$ & $13,8 \mathrm{a}$ & 1656,8 bcd \\
\hline halosulfuron & 112,5 & ALS & $86,0(8,8)$ bc & 30,1 abcd & 10,9 abcd & 1063,7 cde \\
\hline iodosulfuron-methyl & 10,0 & ALS & $4,0(2,0)$ ef & $10,4 \mathrm{f}$ & 9,1 bcd & 693,9 efg \\
\hline metsulfuron-methyl & 2,4 & ALS & $149,0(12,2) \mathrm{a}$ & $31,6 \mathrm{ab}$ & 14,0 a & 1307,2 abcd \\
\hline nicosulfuron & 40,0 & ALS & $0,0(0,7) \mathrm{f}$ & $0,0 \mathrm{~g}$ & $8,5 \mathrm{~cd}$ & $419,9 \mathrm{~g}$ \\
\hline pyrithiobac-sodium & 140,0 & ALS & $121,0(10,5) \mathrm{ab}$ & $31,0 \mathrm{ab}$ & $12,6 \mathrm{ab}$ & $1540,9 \mathrm{ab}$ \\
\hline trifloxysulfuron-sodium & 22,5 & ALS & $0,0(0,7) \mathrm{f}$ & $0,0 \mathrm{~g}$ & $7,9 \mathrm{~d}$ & $529,0 \mathrm{fg}$ \\
\hline 2,4-D & 720,0 & Auxina & $138,0(11,0) \mathrm{ab}$ & $35,0 \mathrm{ab}$ & $14,3 \mathrm{a}$ & $1264,0 \mathrm{bcd}$ \\
\hline quinclorac & 375,0 & Auxina & $117,0(10,8) a b$ & $34,0 \mathrm{ab}$ & $14,6 \mathrm{a}$ & 1717,6 a \\
\hline atrazina & $1.250,0$ & Foto II & $97,0(9,7) \mathrm{ab}$ & $21,1 \mathrm{e}$ & $13,8 \mathrm{a}$ & 1429,9 abc \\
\hline bentazon & 600,0 & Foto II & $162,0(12,7) \mathrm{a}$ & 28,9 abcde & 11,1 abcd & $1514,0 \mathrm{ab}$ \\
\hline linuron & $1.350,0$ & Foto II & $127,0(11,2) a b$ & 30,6 abc & 11,4 abcd & $1439,1 \mathrm{abc}$ \\
\hline fomesafen & 187,5 & Protox & $123,0(11,1) \mathrm{ab}$ & $30,4 \mathrm{abc}$ & $11,8 \mathrm{abcd}$ & $1261,9 \mathrm{bcd}$ \\
\hline lactofen & 120,0 & Protox & $153,0(12,1)$ a & 29,0 abcde & $13,3 \mathrm{a}$ & $1671,8 \mathrm{ab}$ \\
\hline oxadiazon & 600,0 & Protox & $130,0(11,3) \mathrm{ab}$ & 28,3 abcde & 12,0 abc & 1308,1 abcd \\
\hline oxyfluorfen & 720,0 & Protox & $97,0(9,6)$ ab & 27,0 bcde & 13,3 a & $1518,4 \mathrm{ab}$ \\
\hline $\mathrm{F}_{\text {Tratamento }}$ & & & $15,425^{* *}$ & $13,581 * *$ & $1,683^{\mathrm{ns}}$ & $5,495^{* *}$ \\
\hline $\mathrm{F}_{\text {Bloco }}$ & & & $1,326^{\mathrm{ns}}$ & $1,244^{\mathrm{ns}}$ & $1,194^{\mathrm{ns}}$ & $2,479^{\mathrm{ns}}$ \\
\hline CV $(\%)$ & & & 25,06 & 26,11 & 23,82 & 23,64 \\
\hline d.m.s. & & & 3,029 & 8,991 & 4,088 & 430,077 \\
\hline
\end{tabular}

1/ Adicionou-se o adjuvante Assist $\left(0,5 \% \mathrm{v} \mathrm{v}^{-1}\right)$ à calda de aplicação. ${ }^{2 /}$ dados originais, entre parênteses, foram transformados em $(\mathrm{x}+0,5)^{0,5}$ Médias seguidas de mesma letra, nas linhas, não diferem estatisticamente entre si a $5 \%$ de probabilidade pelo teste LSD.

${ }^{* *}$ significativo a $1 \%$ de probabilidade; * significativo a $5 \%$ de probabilidade; ${ }^{\text {ns }}$ não significativo.

oxadiazon e oxyfluorfen, que proporcionaram intoxicação severa no período entre 7 e 26 DAA, também não reduziram a massa seca da parte aérea da grama. Provavelmente, após a metabolização dos herbicidas, houve rápida disponibilização das reservas de energia para o restabelecimento do desenvolvimento normal da grama, caracterizando um efeito compensatório no acúmulo de biomassa após o desaparecimento das injúrias. Assim, ressaltase a importância da realização de mais estudos para identificar e descrever os mecanismos fisiológicos envolvidos nos processos de desintoxicação dos herbicidas em plantas.

Akanda et al. (1997), avaliando o controle químico de Solanum viarum e $P$. notatum, observaram sintomas leves $(\leq 2,0 \%)$ provocados pelo herbicida 2,4-D (2.500 $\left.\mathrm{g} \mathrm{ha}^{-1}\right)$ por até $35 \mathrm{DAA}$, enquanto para fomesafen $\left(1.000 \mathrm{~g} \mathrm{ha}^{-1}\right) \mathrm{e}$ lactofen (1.200 $\left.\mathrm{g} \mathrm{ha}^{-1}\right)$ verificaram-se sintomas severos $(\geq 25,0 \%)$ por até 145 DAA.
De acordo com Willard et al. (1990), a aplicação do sethoxydim (280 $\left.\mathrm{g} \mathrm{ha}^{-1}\right)$ e do fluazifop-p-butil (280 $\mathrm{g} \mathrm{ha}^{-1}$ ) reduziu a massa seca da grama $P$. notatum em torno de 33,0 e $38,9 \%$, respectivamente, aos 12 DAA.

Na Tabela 4 são apresentadas as porcentagens de intoxicação em plantas de A. compressus após a aplicação de diferentes herbicidas. Pode-se observar que aos 3 DAA somente a atrazina não promoveu intoxicação no gramado, enquanto o herbicida oxyfluorfen apresentou sintomas severos, na ordem de $37,5 \%$. Os demais herbicidas proporcionaram sintomas variando entre 1,8 e $5,0 \%$ para a mesma data de avaliação.

Já aos 7 DAA, os inibidores da ACCase mostraram resultados semelhantes aos verificados na grama $P$. notatum, promovendo sintomas severos e com valores superiores a 35,0 e $53,8 \%$ para os herbicidas fluazifop-p- 
butil e sethoxydim, respectivamente. Entretanto, diferentemente do observado na grama $P$. notatum, os sintomas severos proporcionados por esses herbicidas em $A$. compressus apresentaram duração de apenas 14 DAA.

Dos inibidores da ALS que apresentaram sintomas de intoxicação leves por até 14 DAA, destacam-se chlorimuron-ethyl, ethoxysulfuron, halosulfuron e metsulfuron-methyl. Os herbicidas iodosulfuron-methyl, pyrithiobacsodium e trifloxysulfuron-sodium apresentaram sintomas de moderados a severos aos 7 DAA, regredindo para sintomas leves a partir de 14 DAA. Já os herbicidas bispyribac-sodium e nicosulfuron proporcionaram intoxicação severa (> 20,0\%), persistindo os sintomas por até 14 DAA.

Os mimetizadores de auxina 2,4-D e quinclorac destacaram-se entre os herbicidas que apresentaram médias leves de toxicidade, com o desaparecimento dos sintomas por completo a partir de 14 DAA. Ressalta-se que o quinclorac prejudicou severamente o aspecto visual da grama $P$. notatum por até 26 DAA (Tabela 2).

Com relação aos inibidores do fotossistema II, os herbicidas atrazina e bentazon apresentaram sintomas de intoxicação leves por apenas 14 DAA. Contudo, o linuron mostrou toxicidade severa aos 7 DAA e moderada a partir de 14 DAA.

Para os inibidores da Protox, o fomesafen foi o herbicida que mais se destacou, uma vez que proporcionou intoxicação inferior a 5,3\% por no máximo 14 DAA. Os sintomas proporcionados pelo oxyflourfen em A. compressus foram considerados severos e persistiram por até 14 DAA.

O lactofen e o oxadiazon promoveram sintomas severos aos 7 DAA; a partir desse período até os 14 DAA a toxicidade foi moderada, com desaparecimento total dos sintomas visuais a partir de 26 DAA.

Tabela 4 - Porcentagem de intoxicação de plantas de A. compressus, após a aplicação de diferentes herbicidas. Botucatu-SP, 2006

\begin{tabular}{|c|c|c|c|c|c|c|c|}
\hline \multirow{2}{*}{ Tratamento } & \multirow{2}{*}{$\begin{array}{c}\text { Dose } \\
\left.\left(\text { g i.a. }^{-1}\right)^{-1}\right)\end{array}$} & \multirow{2}{*}{$\begin{array}{c}\text { Mecanismo } \\
\text { de ação }\end{array}$} & \multicolumn{5}{|c|}{ Dias após a aplicação ${ }^{\underline{2} /}$} \\
\hline & & & 3 & 7 & 14 & 26 & 49 \\
\hline Testemunha & $\overline{---}$ & $\overline{---}$ & $0,0(0,0) \mathrm{f}$ & $0,0(0,0) \mathrm{i}$ & $0,0(0,0) \mathrm{j}$ & $\overline{0,0}$ & 0,0 \\
\hline fluazifop-p-butil & 125,0 & Accase & $4,0(11,0)$ de & $35,0(35,9) \mathrm{cd}$ & $53,8(47,2) \mathrm{a}$ & 0,0 & 0,0 \\
\hline sethoxydim ${ }^{\frac{1}{}}$ & 276,0 & Accase & $3,5(10,8) \mathrm{de}$ & $53,8(47,1)$ ab & $45,0(42,1)$ abc & 0,0 & 0,0 \\
\hline bispyribac-sodium & 25,0 & ALS & $5,0(12,7) \mathrm{cd}$ & $46,3(42,8)$ bc & $40,0(39,1) b c$ & 0,0 & 0,0 \\
\hline chlorimuron-ethyl & 15,0 & ALS & $2,3(8,4) \mathrm{de}$ & $3,0(9,9)$ fghi & $2,3(8,1)$ hi & 0,0 & 0,0 \\
\hline ethoxysulfuron & 150,0 & ALS & $2,0(7,9)$ de & $3,8(10,1)$ fghi & $1,5(6,9) \mathrm{ij}$ & 0,0 & 0,0 \\
\hline halosulfuron & 112,5 & ALS & $2,0(8,0) \mathrm{de}$ & $3,5(8,7)$ ghi & $6,0(13,0)$ efghi & 0,0 & 0,0 \\
\hline iodosulfuron-methyl & 10,0 & ALS & $3,8(10,7) \mathrm{de}$ & $13,3(21,3) \mathrm{e}$ & $4,0(11,5)$ fghi & 0,0 & 0,0 \\
\hline metsulfuron-methyl & 2,4 & ALS & $3,3(10,3) \mathrm{de}$ & $8,3(15,6)$ efgh & $6,0(13,0)$ efghi & 0,0 & 0,0 \\
\hline nicosulfuron & 40,0 & ALS & $4,8(12,3) \mathrm{d}$ & $48,8(44,3)$ bc & $48,8(44,3) \mathrm{ab}$ & 0,0 & 0,0 \\
\hline pyrithiobac-sodium & 140,0 & ALS & $2,8(9,3) \mathrm{de}$ & $11,8(19,3)$ ef & $3,3(9,9)$ ghi & 0,0 & 0,0 \\
\hline trifloxysulfuron-sodium & 22,5 & ALS & $5,0(12,8) \mathrm{cd}$ & $35,0(35,8) \mathrm{cd}$ & $9,5(17,4)$ efg & 0,0 & 0,0 \\
\hline $2,4-\mathrm{D}$ & 720,0 & Auxina & $2,3(8,6) \mathrm{de}$ & $1,3(6,3)$ hi & $4,3(11,2)$ fghi & 0,0 & 0,0 \\
\hline quinclorac & 375,0 & Auxina & $1,8(7,5) \mathrm{de}$ & $5,0(12,4)$ efgh & $7,5(15,7)$ efgh & 0,0 & 0,0 \\
\hline atrazina & $1.250,0$ & Fotossistema II & $0,0(0,0) \mathrm{f}$ & $9,0(17,1)$ efg & $7,0(14,5)$ efghi & 0,0 & 0,0 \\
\hline bentazon & 600,0 & Fotossistema II & $1,8(7,5) \mathrm{de}$ & $2,8(9,3)$ fghi & $6,8(14,9)$ efgh & 0,0 & 0,0 \\
\hline linuron & $1.350,0$ & Fotossistema II & $2,8(9,3) \mathrm{de}$ & $38,8(38,3) \mathrm{bcd}$ & $18,8(25,3) \mathrm{d}$ & 0,0 & 0,0 \\
\hline fomesafen & 187,5 & Protox & $2,3(8,1) \mathrm{de}$ & $5,3(12,8)$ efgh & $2,5(8,7) \mathrm{hi}$ & 0,0 & 0,0 \\
\hline lactofen & 120,0 & Protox & $11,8(18,4) \mathrm{c}$ & $28,8(32,2) \mathrm{d}$ & $10,5(18,7)$ def & 0,0 & 0,0 \\
\hline oxadiazon & 600,0 & Protox & $18,8(25,3) b$ & $28,8(32,4) \mathrm{d}$ & $11,3(19,5) \mathrm{de}$ & 0,0 & 0,0 \\
\hline oxyfluorfen & 720,0 & Protox & $37,5(37,5) \mathrm{a}$ & $68,8(56,8)$ a & $35,0(35,6) \mathrm{c}$ & 0,0 & 0,0 \\
\hline $\mathrm{F}_{\text {Tratamento }}$ & & & $15,824 * *$ & $19,390^{* *}$ & $25,464^{* *}$ & -- & --- \\
\hline $\mathrm{F}_{\text {Bloco }}$ & & & $0,544^{\mathrm{ns}}$ & $1,111^{\mathrm{ns}}$ & $2,150^{\mathrm{ns}}$ & -- & -- \\
\hline $\mathrm{CV}(\%)$ & & & 36,09 & 30,07 & 27,26 & -- & -- \\
\hline d.m.s. & & & 5,710 & 10,298 & 7,651 & -- & --- \\
\hline
\end{tabular}

1/ Adicionou-se o adjuvante Assist $\left(0,5 \% \mathrm{v} \mathrm{v}^{-1}\right)$ à calda de aplicação. ${ }^{2 /}$ dados originais, entre parênteses, foram transformados em arco seno $(\mathrm{x} / 100)^{0,5}$. Médias seguidas de mesma letra, nas linhas, não diferem estatisticamente entre si a $5 \%$ de probabilidade pelo teste LSD. ** significativo a $1 \%$ de probabilidade; $*$ significativo a $5 \%$ de probabilidade; ${ }^{\text {ns }}$ não significativo. 
Conforme relatado por Johnson \& Carrow (1999), a aplicação em pré-emergência do oxadiazon (3.400 $\mathrm{g} \mathrm{ha}^{-1}$ ) não prejudicou a qualidade estética de oito cultivares de grama Zoysia por até 77 DAA. Em razão de esse herbicida ter sido aplicado em pós-emergência no ensaio realizado com $P$. notatum e A. compressus, pode-se inferir que a aplicação em pré ou pós-emergência pode ser um fator importante para a determinação de seletividade de herbicidas em gramados.

Assim, dos herbicidas avaliados, os que proporcionaram sintomas de intoxicação leves a moderados na grama $A$. compressus por no máximo 14 DAA foram chlorimuron-ethyl, ethoxysulfuron, halosulfuron, iodosulfuronmethyl, metsulfuron-methyl, pyrithiobacsodium, 2,4-D, quinclorac, atrazina, bentazon e fomesafen. Os herbicidas fluazifop-p-butil, sethoxydim, bispyribac-sodium, nicosulfuron, trifloxysulfuron-sodium, linuron, lactofen, oxadiazon e oxyfluorfen foram os que promoveram toxicidade severa $(\geq 20,0 \%)$ entre 7 e 14 DAA. Ressalta-se que, a partir de 26 DAA, os sintomas de intoxicação promovidos pelos herbicidas avaliados na grama $A$. compressus desapareceram completamente.

Na Tabela 5 são apresentados os valores médios de número de inflorescência, altura da planta, altura de inflorescência e massa seca do gramado de $A$. compressus, após a aplicação de diferentes herbicidas, aos 49 DAA.

Verificou-se que fluazifop-p-butil, chlorimuron-ethyl, ethoxysulfuron, iodosulfuronmethyl, pyrithiobac-sodium, trifloxysulfuronsodium, atrazina, fomesafen e lactofen não produziram redução significativa da densidade de inflorescências. Contudo, os demais herbicidas avaliados promoveram redução entre 65,3 e 94,0\% da densidade de inflorescência avaliada aos 49 DAA.

Tabela 5 - Valores médios de número de inflorescência, altura da planta, altura de inflorescência e massa seca do gramado de A. compressus aos 49 dias após a aplicação de diferentes herbicidas. Botucatu-SP, 2006

\begin{tabular}{|c|c|c|c|c|c|c|}
\hline \multirow[t]{2}{*}{ Tratamento } & Dose & $\begin{array}{l}\text { Mecanismo } \\
\text { de acão }\end{array}$ & $\begin{array}{l}\text { Densidade de } \\
\text { Inflorescência }\end{array}$ & $\begin{array}{c}\text { Altura da } \\
\text { inflorescência }\end{array}$ & $\begin{array}{c}\text { Altura da } \\
\text { grama }\end{array}$ & \multirow{2}{*}{$\frac{\text { Massa seca }}{\left(\mathrm{kg} \mathrm{ha}^{-1}\right)}$} \\
\hline & $\left(\right.$ g i.a. ha $\left.^{-1}\right)$ & & $\left(\text { número } \mathrm{m}^{-2}\right)^{2 /}$ & \multicolumn{2}{|c|}{$(\mathrm{cm})$} & \\
\hline Testemunha & --- & --- & $167,0(12,3)$ ab & $14,5 \mathrm{ab}$ & $9,0 \mathrm{ab}$ & $1414,7 \mathrm{a}$ \\
\hline fluazifop-p-butil & 125,0 & Accase & $69,0(8,3)$ bcdef & $14,0 \mathrm{ab}$ & $6,0 \mathrm{c}$ & $432,7 \mathrm{~g}$ \\
\hline sethoxydim $^{\underline{1}}$ & 276,0 & Accase & $30,0(5,0) \mathrm{fg}$ & $9,3 \mathrm{~b}$ & $6,6 \mathrm{bc}$ & 711,9 defg \\
\hline bispyribac-sodium & 25,0 & ALS & $54,0(6,1)$ efg & $14,3 \mathrm{ab}$ & $6,8 \mathrm{abc}$ & 587,3 efg \\
\hline chlorimuron-ethyl & 15,0 & ALS & $127,0(10,8)$ abcd & $16,1 \mathrm{a}$ & $7,5 \mathrm{abc}$ & 1027,2 abcd \\
\hline ethoxysulfuron & 150,0 & ALS & $173,0(12,7) \mathrm{a}$ & $13,4 \mathrm{ab}$ & $7,5 \mathrm{abc}$ & 791,6 cdefg \\
\hline halosulfuron & 112,5 & ALS & $48,0(7,0)$ def & $14,5 \mathrm{ab}$ & $7,9 \mathrm{abc}$ & 987,3 bcde \\
\hline iodosulfuron-methyl & 10,0 & ALS & $155,0(11,8)$ abc & $12,4 \mathrm{ab}$ & $6,3 \mathrm{bc}$ & 992,4 bcd \\
\hline metsulfuron-methyl & 2,4 & ALS & $42,0(6,1)$ efg & $15,8 \mathrm{a}$ & $8,4 \mathrm{abc}$ & 872,3 cdef \\
\hline nicosulfuron & 40,0 & ALS & $10,0(2,6) \mathrm{g}$ & $13,3 \mathrm{ab}$ & $6,3 \mathrm{bc}$ & $390,1 \mathrm{~g}$ \\
\hline pyrithiobac-sodium & 140,0 & ALS & $176,0(12,3) \mathrm{ab}$ & $15,9 \mathrm{a}$ & $8,1 \mathrm{abc}$ & 656,5 defg \\
\hline trifloxysulfuron-sodium & 22,5 & ALS & $103,0(10,0)$ abcde & $12,1 \mathrm{ab}$ & $6,0 \mathrm{c}$ & 706,0 defg \\
\hline 2,4-D & 720,0 & Auxina & $58,0(7,2)$ def & $13,0 \mathrm{ab}$ & $7,8 \mathrm{abc}$ & $853,6 \mathrm{cdef}$ \\
\hline quinclorac & 375,0 & Auxina & $58,0(7,2)$ def & $12,6 \mathrm{ab}$ & $8,4 \mathrm{abc}$ & 839,4 cdef \\
\hline atrazina & 1250,0 & Fotossistema II & $203,0(14,0)$ a & $13,5 \mathrm{ab}$ & $7,5 \mathrm{abc}$ & $1190,4 \mathrm{abc}$ \\
\hline bentazon & 600,0 & Fotossistema II & $51,0(6,9)$ def & $16,8 \mathrm{a}$ & $8,0 \mathrm{abc}$ & 777,5 defg \\
\hline linuron & 1350,0 & Fotossistema II & $55,0(6,7)$ efg & $14,9 \mathrm{a}$ & $8,8 \mathrm{abc}$ & 1000,6 bcd \\
\hline fomesafen & 187,5 & Protox & $141,0(12,0) \mathrm{ab}$ & $16,0 \mathrm{a}$ & $8,6 \mathrm{abc}$ & $1293,5 \mathrm{ab}$ \\
\hline lactofen & 120,0 & Protox & $72,0(8,3)$ bcdef & $13,5 \mathrm{ab}$ & 8,0 abc & $583,3 \mathrm{fg}$ \\
\hline oxadiazon & 600,0 & Protox & $56,0(7,4)$ def & $17,0 \mathrm{a}$ & $9,6 \mathrm{a}$ & 867,5 cdef \\
\hline oxyfluorfen & 720,0 & Protox & $55,0(7,2)$ def & $14,3 \mathrm{ab}$ & $7,5 \mathrm{abc}$ & $471,5 \mathrm{fg}$ \\
\hline $\mathrm{F}_{\text {Tratamento }}$ & & & $4,065^{* *}$ & $0,930^{\mathrm{ns}}$ & $0,998^{\text {ns }}$ & $3,631 * *$ \\
\hline $\mathrm{F}_{\text {Bloco }}$ & & & $4,157^{* *}$ & $1,755^{\mathrm{ns}}$ & $0,386^{\mathrm{ns}}$ & $0,431^{\mathrm{ns}}$ \\
\hline CV $(\%)$ & & & 34,22 & 26,59 & 26,71 & 34,28 \\
\hline d.m.s. & & & 4,192 & 5,317 & 2,886 & 402,846 \\
\hline
\end{tabular}

1/ Adicionou-se o adjuvante Assist $\left(0,5 \% \mathrm{v} \mathrm{v}^{-1}\right)$ à calda de aplicação. ${ }^{2 /}$ dados originais, entre parênteses, foram transformados em $(\mathrm{x}+0,5)^{0,5}$. Médias seguidas de mesma letra, nas linhas, não diferem estatisticamente entre si a $5 \%$ de probabilidade pelo teste LSD.

${ }^{* *}$ significativo a $1 \%$ de probabilidade; ${ }^{*}$ significativo a $5 \%$ de probabilidade; ${ }^{\text {ns }}$ não significativo. 
Com relação à altura das inflorescências, nenhum dos herbicidas avaliados diferiu estatisticamente em relação à testemunha. Entretanto, para a altura da planta, observouse que somente os herbicidas fluazifop-p-butil (ACCase) e o trifloxysulfuron-sodium (ALS) influenciaram o crescimento do gramado, promovendo reduções de até $33,3 \%$, em relação à testemunha, aos 49 DAA.

Dos herbicidas avaliados, apenas chlorimuron-ethyl, atrazina e fomesafen apresentaram produção de massa seca da parte aérea semelhante à da testemunha no período de 49 DAA. Ressalta-se que esses herbicidas promoveram sintomas leves de intoxicação na grama $A$. compressus.

No entanto, os herbicidas ethoxysulfuron, halosulfuron, metsulfuron-methyl, 2,4-D, quinclorac e bentazon, além de promoverem sintomas leves de intoxicação, também proporcionaram redução na massa seca da parte aérea da grama $A$. compressus em cerca de 44,$0 ; 30,2 ; 38,3 ; 39,7 ; 40,7 ;$ e 45,0\%, respectivamente, em relação à testemunha.

Da mesma forma, os herbicidas iodosulfuron-methyl e pyrithiobac-sodium reduziram a massa seca da parte aérea, respectivamente, em 29,8 e $53,6 \%$, porém proporcionaram sintomas moderados de intoxicação na grama A. compressus (Tabelas 4 e 5). Dessa maneira, verifica-se que herbicidas também podem ser utilizados em gramados como reguladores vegetais, com o objetivo de inibir o crescimento vegetativo das plantas, bem como inibir a emissão das inflorescências (Johnson, 1990; Baker et al., 1999).

De acordo com Christoffoleti \& Aranda (2001), a seletividade de herbicidas aos diversos tipos de grama está em função principalmente da espécie de grama e do tipo de herbicida aplicado, bem como da dose utilizada.

Pode-se concluir que, dos herbicidas avaliados no presente estudo, os que apresentaram potencial de seletividade para o gramado de $P$. notatum foram chlorimuron-ethyl, ethoxysulfuron, pyrithiobac-sodium, 2,4-D, bentazon e fomesafen; para a grama $A$. compressus, os herbicidas foram chlorimuron-ethyl, ethoxysulfuron, halosulfuron, iodosulfuron-methyl, metsulfuron-methyl, pyrithiobac-sodium,
2,4-D, quinclorac, atrazina e bentazon, além do fomesafen.

Portanto, esses herbicidas podem ser utilizados no manejo de gramados tanto em programas de controle de plantas daninhas, fornecendo opções de mecanismos de ação distintos para a prevenção de surgimento de plantas resistentes a herbicidas, quanto como reguladores vegetais, com o intuito de reduzir as operações de corte em gramados. Contudo, mais estudos são necessários com relação ao uso desses herbicidas como reguladores vegetais, principalmente no que se refere a doses e momento de aplicação.

\section{LITERATURA CITADA}

AKANDA, R. U. et al. Influence of postemergence herbicides on tropical soda apple (Solanum viarum) and bahiagrass (Paspalum notatum). Weed Technol., v. 11, n. 3, p. 656-661, 1997.

BAKER, R. D. et al. Bahiagrass (Paspalum notatum) seedhead suppression following consective yearly applications of plant growth retardants. Weed Technol., v. 13, n. 2 , p. $378-384,1999$

BRECKE, B. J.; UNRUH, B.; DUSKY, J. A. Torpedograss (Panicum repens) control with quinclorac in bermudagrass (Cynodon dactylon x C. transvaalensis) turf. Weed Technol., v. 15, n. 4 , p. $732-736,2001$

BUSEY, P. Cultural management of weed in turfgrass: a review. Crop Sci., v. 43, n. 6, p. 1899-1911, 2003.

BUSEY, P.; JOHNSTON, D. L. Impact of cultural factors on weed populations in St. Augustinegrass turf. Weed Sci., v. 54, n. 5 , p. $961-967,2006$

CHRISTOFFOLETI, P. J.; ARANDA, A. N. Seletividade de herbicidas a cinco tipos de gramas. Planta Daninha, v. 19, n. 2, p. 273-278, 2001.

FAGERNESS, M. J.; YELVERTON, F. H.; COOPER, R. J. Bermudagrass [Cynodon dactylon (L.) Pers.] and Zoysiagrass (Zoysia japonica) establishment after preemergente herbicide applications. Weed Technol., v. 16, n. 3, p. 597-602, 2002.

FREITAS, F. C. L. et al. Eficiência do triclopyr no controle de plantas daninhas em gramado (Paspalum notatum).

Planta Daninha, v. 21, n. 1, p. 159-164, 2003.

JOHNSON, B. J. Response of bahiagrass (Paspalum notatum) to plant growth regulators. Weed Technol., v. 4, n. 4, p. 895-899, 1990.

Planta Daninha, Viçosa-MG, v. 28, n. 2, p. 365-374, 2010 
JOHNSON, B. J.; CARROW, R. N. Tolerance of zoysiagrass (Zoysia spp.) cultivars to preemergence herbicides. Weed Technol., v. 13, n. 4, p. 706-712, 1999.

McCARTY, L. B.; PORTER, W.; COLVIN, D. L. Sod regrowth of St. Augustinegrass after preemergence herbicide application. Agron. J., v. 87, n. 3, p. 503-507, 1995.

RODRIGUES, B. N.; ALMEIDA, F. S. Guia de herbicidas. 5.ed. Londrina: 2005. 592 p.
SOCIEDADE BRASILEIRA DA CIÊNCIA DAS PLANTAS DANINHAS - SBCPD. Procedimentos para instalação, avaliação e análise de experimentos com herbicidas. Londrina: 1995. $42 \mathrm{p}$.

TURNER, D. L.; SHARPE, S. S.; DICKENS, R. Herbicide effects on tensile strength and rooting of centipedegrass sod Hortscience, v. 25, n. 5, p. 541-544, 1990

WILLARD, T. R.; PEACOCK, C. M.; SHILLING, D. G. Photosynthesis as an index of turfgrass growth following application of herbicides. Hortscience, v. 25, n. 4 , p. 451-453, 1990. 